\title{
Graphene Oxide-Induced pH Alteration, Iron Overload, and Subsequent Oxidative Damage in Rice (Oryza sativa L.): A New Mechanism of Nanomaterial Phytotoxicity
}

\author{
Peng Zhang,* Zhiling Guo, Wenhe Luo, Fazel Abdolahpur Monikh, Changiian Xie, \\ Eugenia Valsami-Jones, Iseult Lynch, and Zhiyong Zhang*
}

Cite This: Environ. Sci. Technol. 2020, 54, 3181-3190

Read Online

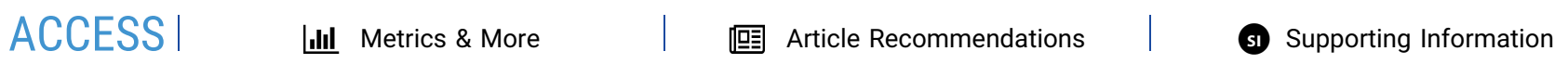

ABSTRACT: The mechanism of graphene-based nanomaterial (GBM)-induced phytotoxicity and its association with the GBM physicochemical properties are not yet fully understood. The present study compared the effects of graphene oxide (GO) and reduced GO (rGO) on rice seedling growth under hydroponic conditions for 3 weeks. GO at 100 and $250 \mathrm{mg} / \mathrm{L}$ reduced shoot biomass (by 25 and 34\%, respectively) and shoot elongation (by 17 and $43 \%$, respectively) and caused oxidative damage, while rGO exhibited no overt effect except for the enhancement of the antioxidant enzyme activities, suggesting that the surface oxygen content is a critical factor affecting the biological impacts of GBMs. GO treatments (100 and $250 \mathrm{mg} / \mathrm{L}$ ) enhanced the iron $(\mathrm{Fe})$

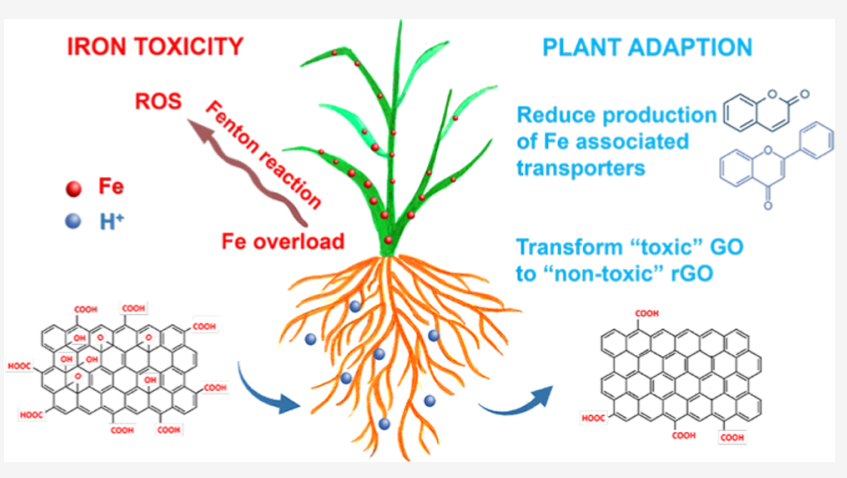
translocation and caused excessive Fe accumulation in shoots $(2.2$ and 3.6 times higher than control), which was found to be the main reason for the oxidative damage in shoots. GO-induced acidification of the nutrient solution was the main driver for the Fe overload in plants. In addition to the antioxidant regulators, the plants triggered other pathways to defend against the Fe toxicity via downregulation of the Fe transport associated metabolites (mainly coumarins and flavonoids). Plant root exudates facilitated the reduction of toxic GO to nontoxic rGO, acting as another route for plant adaption to GO-induced phytotoxicity. This study provides new insights into the mechanism of the phytotoxicity of GBMs. It also provides implications for the agricultural application of GBM that the impacts of GBMs on the uptake of multiple nutrients in plants should be assessed simultaneously and reduced forms of GBMs are preferential to avoid toxicity.

\section{INTRODUCTION}

Graphene is a two-dimensional carbon-based nanomaterial composed of a single layer of $\mathrm{sp}^{2}$ hybridized carbon atoms. ${ }^{1}$ It is considered as one of the most promising engineered nanomaterials (ENMs) with the potential to be used in various sectors such as electronics, medical, energy, and environment, ${ }^{2-4}$ due to its unique electronic, thermal, and mechanical properties. The increasing production and use of graphenebased materials (GBMs) will inevitably increase the likelihood of their release into the environment, and thus their potential adverse impacts on environmental and human safety need to be fully assessed. ${ }^{5}$

There have been extensive studies regarding the toxicity of GBMs on cells and microorganisms, ${ }^{6,7}$ while knowledge of the potential impacts of GBMs on the growth of higher plants is still lacking. There is concern that GBMs may affect plant growth and/or accumulate in crops or vegetables, ${ }^{8}$ causing potential risks to human health. Recent studies showed that GBMs have the potential to be used as a carrier for fertilizers to enable the slow release of nutrients and thus enhance the nutrient use efficiency by plants; ${ }^{9-11}$ however, such applica- tions in real agriculture are not currently pursued partially due to the concerns over the potential adverse impacts of GBMs on the overall agricultural ecosystem, including soil functioning (e.g., bacterial community, enzyme activity) ${ }^{12-14}$ the potential trophic transfer of GBMs, ${ }^{15}$ and cumulative effects of GBMs after repeated application and over multiple growing seasons.

Overt toxicity of GBMs to plant such as inhibition of biomass production and shoot or root elongation have been reported at high exposure concentrations. ${ }^{16,17}$ However, a number of studies also reported that GBMs induced physiological alterations (e.g., hormone levels, nutrient uptake) $)^{17,18}$ or oxidative stress (e.g., enhanced antioxidant enzymatic activities, lipid peroxidation, or $\mathrm{H}_{2} \mathrm{O}_{2}$ overaccumu-

Received: September 25, 2019

Revised: January 4, 2020

Accepted: February 21, 2020

Published: February 21, 2020 
lation $)^{16,19}$ even at environmentally relevant concentrations $(0.01-1 \mathrm{mg} / \mathrm{L}){ }^{20}$ suggesting that subtle physiological processes are more sensitive indices than apparent toxicity indices (e.g., biomass, root/shoot length) for evaluating the phytotoxicity of GBMs. ${ }^{21}$ In addition to the negative effects, positive effects resulting from exposure to GBMs on plant growth are also reported. For example, hydrated graphene ribbons promoted the germination of wheat seeds, upregulated carbohydrate, amino acids, and fatty acid metabolism during germination and enhanced the tolerance of seeds to oxidative stress. $^{22}$ Due to the hydrophilic nature of GO, it can act as a water transporter to promote seed germination. ${ }^{23}$

There are several reasons that may contribute to the inconsistent reports regarding the phytotoxicity of GBMs. First, phytotoxicity of ENMs is species-dependent, ${ }^{24}$ crossspecies comparison is not always suitable. Second, using different culture media such as soil, ${ }^{23}$ agar, $^{25}$ and hydroponic solutions, ${ }^{1919}$ which have different compositions, can affect the behavior, fate, and toxicity of the GBMs. GBM in soil and agar media usually have low mobility and accessibility to plants, thereby lowering their impact on plant growth found in such media when compared with those observed in hydroponic media. However, this might be not always true. For example, $\mathrm{CeO}_{2}$ ENMs were reported to be more toxic to Lactuca plants in agar medium than in water, which is because Lactuca plants are more sensitive in agar than in water to the toxicity of $\mathrm{Ce}^{3+}$ ions released from $\mathrm{CeO}_{2}$ ENMs. ${ }^{26}$ Lastly, the physicochemical properties of the GBMs used in the previous studies are very diverse and are not fully described in many cases. In reviewing studies on the effects of GBMs on higher plants (Table S1), more than half of the papers considered did not provide sufficient characterization data including lateral size, thickness, and surface oxygen content, which are critical characteristics determining the biological effects of GBMs. ${ }^{5}$ Where provided, the given properties were very varied: the lateral size used in these studies ranged from $30 \mathrm{~nm}$ to $6.5 \mu \mathrm{m}$, the layer thickness ranged from 0.3 to $3.5 \mathrm{~nm}$, and the surface oxygen contents ranged from 3.51 to $38.8 \%$. Clearly, more studies are required to provide sufficient data for cross-comparison and elucidating the mechanism(s) of action of GBMs and to determine the ranges of GBMs' properties that can be used safely to enhance plant growth and/or soil quality and nutrient cycling.

Common forms of graphene, including graphene (G), graphene oxide (GO), and reduced graphene oxide (rGO), are distinct in their surface oxygen contents. GO is the oxidized form of graphene, which contains abundant functional groups including carboxyl, hydroxyl, epoxy, and carbonyl groups. ${ }^{27}$ These functional groups endow GO with high water dispersity and can be used for further functionalization of $\mathrm{GO}$ for different applications. ${ }^{3}$ Previous studies have suggested that GO and rGO have distinct antibacterial activities, ${ }^{28}$ which is attributed to the different modes of interaction of GO and rGO with cell membranes. We hypothesize that comparing the phytotoxicity of GO and rGO, which is yet to be studied, will allow the acquisition of a mechanistic understanding of the actions of GBMs in plants. To do so, we investigated the impacts of $\mathrm{GO}$ and $\mathrm{rGO}$ on the growth of rice plants. Oxidative stress, perturbation of the uptake of macro- and microelements in plants, metabolic alteration, and the transformation of GO and rGO in rice plants were compared to explore the mechanisms of the interaction of $\mathrm{GO}$ and $\mathrm{rGO}$ with plants and their consequences for plant health.

\section{MATERIALS AND METHODS}

2.1. Chemicals and Seeds. GO and rGO were purchased from Chengdu Organic Chemicals Co. Ltd. (Chengdu, China). Morphology, lateral size, height, chemical structure, $\zeta$ potential, and hydrodynamic sizes of GO and rGO were characterized, details of which are described in the Supporting Information (Section 1). All other commercial chemicals were purchased from Sinopharm Chemical Reagent Co., Ltd. (Shanghai, China). Rice (Oryza sativa L.) seeds were purchased from the Chinese Academy of Agricultural Sciences.

2.2. Plant Culture and Exposure. Rice seeds were germinated in the dark for 5 days after sterilization with $10 \%$ $\mathrm{NaClO}$. Uniform seedlings were then selected and each seedling was anchored by a plastic foam with a hole and transferred into a $250 \mathrm{~mL}$ beaker containing $100 \mathrm{~mL}$ of modified 1/4 strength Hoagland solution. All of the beakers were wrapped with black plastic bags to simulate the dark environment in soil. Six replicates were set for each treatment. The seedlings were allowed to grow in a growth chamber (PRX-450C, Saifu, China) with a day/night temperature of $28 / 20{ }^{\circ} \mathrm{C}$, day/night humidity of $50 / 70 \%$, and a $14 \mathrm{~h}$ photoperiod for 10 days before treatment. GO and rGO were then added into a freshly prepared nutrient solution to obtain suspensions with concentrations of $5,50,100$, and $250 \mathrm{mg} / \mathrm{L}$ followed by ultrasonic pretreatment for $10 \mathrm{~min}$. The seedlings were then exposed to the GO and rGO suspensions and allowed to grow for 3 weeks. The suspensions were replenished to $100 \mathrm{~mL}$ with a fresh nutrient solution every 2 days.

2.3. Biomass Production, Seedling Length, and Nutrient Content. After 3 weeks of exposure to the GBMs, rice seedlings were gently lifted from the suspensions, and the roots were rinsed with deionized water repeatedly. GO and rGO that were attached to the roots were rinsed off with deionized water for further analysis. Residual GO and rGO in the beakers were also collected for characterization. The roots and shoots were separated and blotted with clean tissues, and the fresh weights were measured immediately. The seedlings were then lyophilized, and the dry weights of the roots and shoots were measured. To quantify the nutrient content $(\mathrm{Fe}$, $\mathrm{Cu}, \mathrm{Mn}, \mathrm{Zn}, \mathrm{K}, \mathrm{Ca}, \mathrm{Mg}, \mathrm{P}$ ) in plants, dried roots and shoots were ground into fine powders and digested with a 3:1 mixture of $\mathrm{HNO}_{3}$ and $\mathrm{H}_{2} \mathrm{O}_{2}$ on a heating plate $\left(80{ }^{\circ} \mathrm{C}\right.$ for $1 \mathrm{~h}, 120{ }^{\circ} \mathrm{C}$ for $3 \mathrm{~h}$, and $160^{\circ} \mathrm{C}$ for $0.5 \mathrm{~h}$ ). Elemental concentrations in the digestion solution were then analyzed by inductively coupled plasma optical emission spectroscopy (ICP-OES, Thermo,). Multielement standard solutions $(0.5-50 \mathrm{mg} / \mathrm{L})$ containing all of the selected elements were used for external calibration. Blanks were analyzed between every six samples. Spiking recovery experiments and analysis of certified reference material (GBW 07602 Bush Branches and Leaves) were performed for analytical method validation. Recoveries and detection limits for all of the elements are reported in Table S2.

2.4. Stress Response of Rice to GO and rGO. Fresh roots and shoots were excised, homogenized with cold phosphate-buffered saline (PBS) (50 $\mathrm{mM}, \mathrm{pH} 7.8$ ), and centrifuged at $10000 \mathrm{~g}$ and $4{ }^{\circ} \mathrm{C}$ for $10 \mathrm{~min}$. The supernatants were collected for analyses of superoxide dismutase (SOD) and peroxidase (POD) activities and malondialdehyde (MDA) content using assay kits purchased from Nanjing Jiancheng Bioengineering Institute (Nanjing, China). Reactive oxygen species (ROS) accumulation in roots and leaves was examined 
by a DCFH-DA staining method. Fresh leaves and roots were excised and incubated in DCFH-DA (10 mM in PBS) for $2 \mathrm{~h}$ followed by rinsing with PBS three times. ROS accumulation was imaged on a fluorescence microscope (Olympus IX70) with an ex/em of $485 / 522 \mathrm{~nm}$. QA/QC for the assays is described in Section 1, Supporting Information.

2.5. Characterization of GO and rGO after Interaction with Plants. After harvesting of the plants, GO and rGO that were attached to the root surface were washed off from the roots using $\mathrm{ddH}_{2} \mathrm{O}$ (named "GO-W and rGO-W") and collected by centrifugation $(10000 \mathrm{~g}, 30 \mathrm{~min})$. The pellets were then rinsed with hydrochloric acid and ethanol repeatedly to remove salts and organic components. ${ }^{29}$ The obtained pellets were then freeze-dried for analysis. The residual solutions in the beaker after the removal of the plants were also collected and rinsed by the same procedure described above. GO and rGO incubated in the nutrient solution for 3 weeks without the presence of plants were also collected for comparison. All of the materials described above (washed and residual) were analyzed by Raman (Horiba Scientific, Japan), Fourier transform infrared (FTIR) (Bruker Tensor 27 spectrometer, Germany), UV-vis spectroscopy (Purkinje General, Beijing), and X-ray photoelectron spectroscopy (XPS) (ESCALAB 250Xi, Thermo Scientific). To analyze $\mathrm{GO}$ and $\mathrm{rGO}$ on the root surface in situ, fresh root apexes were freeze-dried and analyzed on a Raman spectrometer (Horiba Scientific, Japan). Fresh root apexes were also excised, fixed, and sectioned for transmission electron microscopy (TEM) observation (see details in the Supporting Information).

2.6. Xylem Sap Collection and Fe Concentration Analysis. Rice seedlings were exposed to GO and rGO for 3 weeks as per the exposure procedure in Section 2.2 and then cut off at $2 \mathrm{~cm}$ above the root-shoot interface. The cut surface of the shoot was cleaned with deionized (DI) water and then a silicon tube was fit to the stump (Figure S1). The xylem sap was collected after $24 \mathrm{~h}$ with a pipette and digested in $\mathrm{HNO}_{3}$ $(70 \%)$. Iron concentrations in the xylem saps were analyzed by ICP-OES (Thermo).

2.7. Metabolomics Analysis of Rice Leaves. The fresh rice leaves were thoroughly rinsed with $\mathrm{ddH}_{2} \mathrm{O}$ after harvest and ground into powder in liquid nitrogen. For each sample, $100 \mathrm{mg}$ of the powder was transferred to a $1.5 \mathrm{~mL}$ Eppendorf tube and mixed with $2 \mathrm{~mL}$ of methanol by vortexing vigorously. Samples were ultrasonicated for $1 \mathrm{~h}$ at $4{ }^{\circ} \mathrm{C}$ and dried under a stream of $\mathrm{N}_{2}$. Then, $500 \mu \mathrm{L}$ of cold methanol was added to each sample. The samples were mixed by vigorous vortexing followed by centrifugation at $12000 \mathrm{rpm}$ for $10 \mathrm{~min}$ at $4{ }^{\circ} \mathrm{C}$. A $300 \mu \mathrm{L}$ aliquot of the supernatant was then transferred into a glass sampling vial for analysis. Samples were then analyzed by liquid chromatography-tandem MS (LC-MS/MS). Details of the measurement and data analysis are described in the Supporting Information.

2.8. Data Processing. All statistical data were presented as means \pm standard deviation. Statistical analysis was performed using IBM SPSS Statistics 19. One way analysis of variance (ANOVA) with a Tukey's test was applied after testing the data for normality and homoscedasticity to analyze whether there were significant differences for the data of biomass, root length, stress response, and elemental concentrations between exposure conditions. $P<0.05$ was considered statistically significant.

\section{RESULTS AND DISCUSSION}

3.1. Characterization of GO and rGO. Scanning electron microscopy (SEM) images show the morphology of GO and rGO sheets (Figure 1A,B). The average sizes of GO $(0.089 \pm$
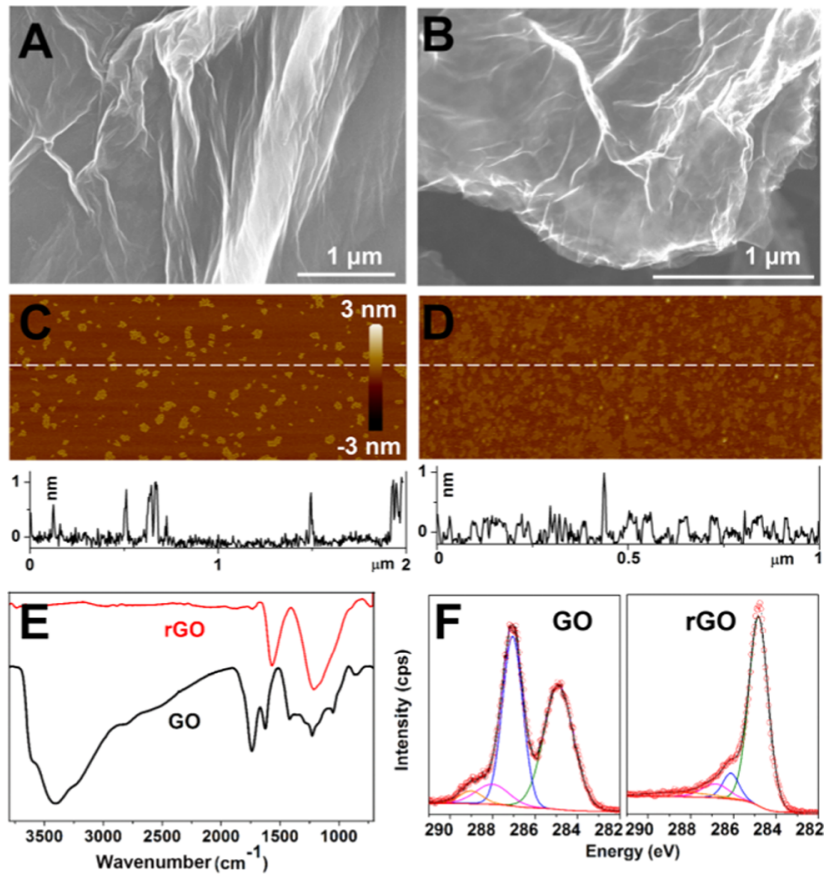

Figure 1. Characterization of GO and rGO. SEM images of GO (A) and rGO (B); AFM images and height profiles of GO (C) and rGO (D); FTIR spectra of GO and rGO (E); XPS spectra of GO and rGO (F).

$0.023 \mu \mathrm{m})$ and $\mathrm{rGO}(0.078 \pm 0.034 \mu \mathrm{m})$ are not significantly different (Figure 1C,D). The size distributions are shown in Figure S2. Atomic force microscopy (AFM) height profiles show that GO and rGO sheets have a thickness of $0.78 \pm 0.26$ and $0.44 \pm 0.23 \mathrm{~nm}$, respectively. FTIR spectra confirm that GO has a significantly higher amount of oxygen-containing groups $\left(\mathrm{O}-\mathrm{H}\right.$ group at $3400 \mathrm{~cm}^{-1}, \mathrm{C}=\mathrm{O}$ group at $1726 \mathrm{~cm}^{-1}$, $\mathrm{C}-\mathrm{O}$ group at 1416 and $1052 \mathrm{~cm}^{-1}$ ) than rGO. XPS survey analysis shows 34.6 and $7.8 \%$ of atomic oxygen in GO and rGO, respectively (Figure S3). Peak fitting analysis of highresolution XPS spectra suggests that the amount of oxygencontaining groups in $\mathrm{GO}$ and $\mathrm{rGO}$ are 61 and 23\%, respectively. DLS analysis suggests that $\mathrm{rGO}$ shows a positive charge in both water and nutrient solution, while GO is negatively charged (Table S3). The hydrodynamic diameters of GO and rGO were larger in the nutrient solution (1398 \pm 347 and $1599 \pm 368 \mathrm{~nm})$ than in deionized water $(1194 \pm 123$ and $865 \pm 98 \mathrm{~nm}$ ), indicating agglomeration of GO and rGO in nutrient solution, and the sizes of GO and rGO in nutrient solution were similar. The high salinity of the nutrient solution contributed to the compression of the double electric layer on the surface of nanomaterials and subsequent aggregation. ${ }^{30}$

3.2. GO and rGO Showed Distinct Effects on Rice Seedling Growth. As shown in Figure 2, GO and rGO showed distinct impacts on the seedling growth of rice plants. GO showed no effect on root elongation after 3 weeks of dosing but significantly reduced the shoot length by $11 \%$ at $100 \mathrm{mg} / \mathrm{L}$ and by $40 \%$ at $250 \mathrm{mg} / \mathrm{L}$ (Figure $2 \mathrm{~A}$ ). GO at 100 and $250 \mathrm{mg} / \mathrm{L}$ also reduced the fresh biomass of both roots 

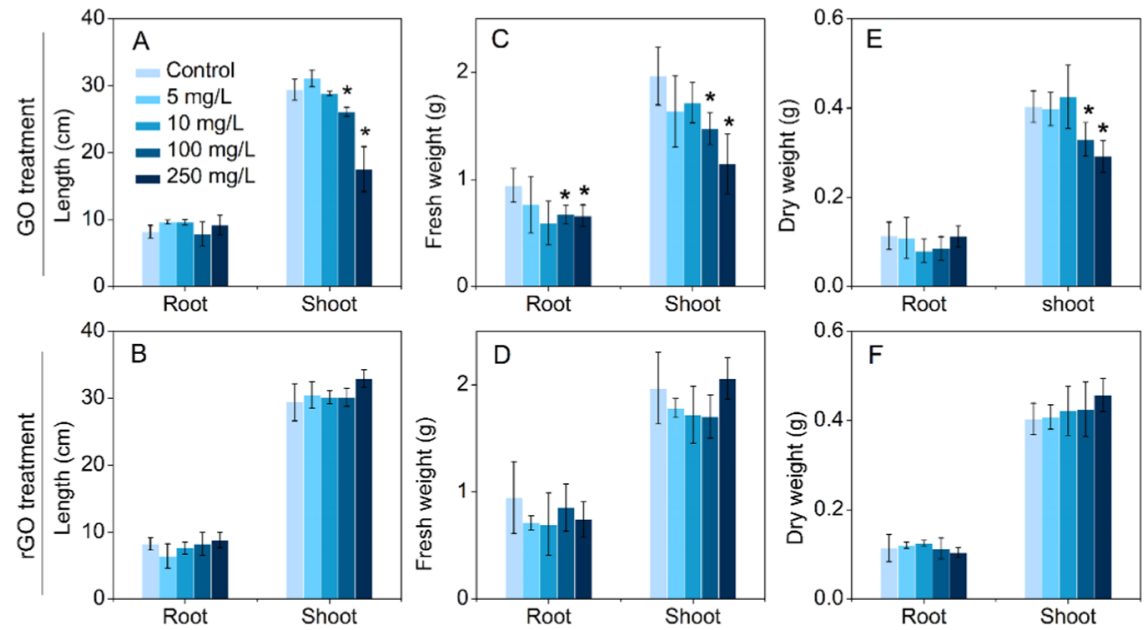

Figure 2. Seedling lengths (A, B), fresh weights (C, D), and dry weights (E, F) of rice seedlings after exposure to different concentrations of GO and rGO for 3 weeks. Top row shows GO treatments and the bottom row shows rGO treatments. * indicates a significant difference compared with control at $P<0.05$.
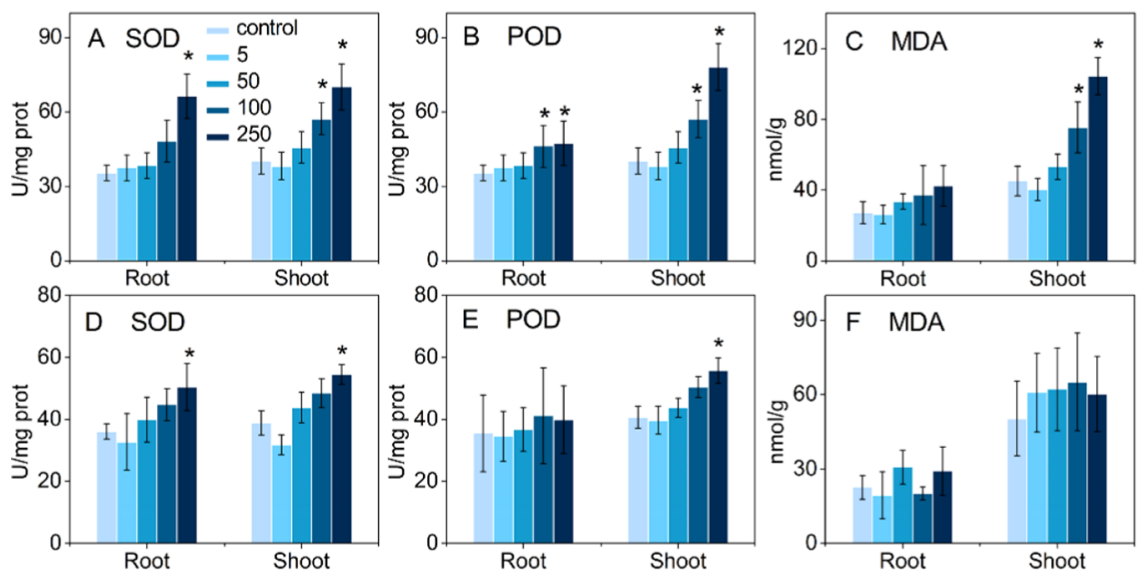

Figure 3. SOD (A, D) and POD (B, E) activities and MDA (C, F) contents in rice after exposure to GO and rGO for 3 weeks. Top row: GO treatments, bottom row: rGO treatments. * indicates significant difference compared with control at $P<0.05$.

and shoots (Figure 2C) and the dry weight of shoots at 100 and $250 \mathrm{mg} / \mathrm{L}$ (Figure $2 \mathrm{E}$ ). In contrast, rGO showed no effect on seedling elongation and biomass production at all concentrations (Figure 2B,D,F).

Since the lateral size and hydrodynamic size of GO and $\mathrm{rGO}$ are not significantly different, they are not related to the different toxicity between GO and rGO. The thicknesses of GO and rGO are slightly different. It has been reported that increasing the thickness would decrease the sharpness of the edge, thus weakening the "nanoknife" effect, ${ }^{31}$ that is, GO with a bigger thickness should show lower effects on plant growth than rGO. However, our result is the opposite, suggesting that thickness is also not a determining factor. These indicate that the phytotoxicity of GBMs is mainly dependent on their surface oxygen content.

3.3. Oxidative Stress Responses Induced by GO and rGO. To further explore the underlying mechanisms of the different responses of rice seedlings and plants to GO and rGO, we examined the oxidative stress responses of rice seedlings to GO and rGO exposure (Figure 3). The activities of antioxidant enzymes including SOD (Figure 3A) and POD (Figure 3B) in shoots following GO treatment were significantly enhanced at 100 and $250 \mathrm{mg} / \mathrm{L}$, while the MDA contents in shoots increased by 37 and $70 \%$ (Figure 3C), respectively. No obvious changes of SOD, POD, and MDA were observed in roots. In rGO treatments, SOD and POD activity only increased at the highest exposure concentration $(250 \mathrm{mg} / \mathrm{L})$ (Figure 3D,E), and there was no alteration of MDA content in either roots or shoots (Figure 3F). Significant overproduction of ROS was found in shoots with GO treatment, while no obvious change was found with rGO treatment (Figure S4). The enhanced activities of antioxidant enzymes represent a defense mechanism of plants against ambient stress. Both GO and rGO triggered the stress response of plants, with the enzymatic antioxidant system failing to protect the plants against GO exposure, with the evidence showing that ROS and MDA over accumulated in GO-exposed plants. Notably, the most overt overaccumulation of MDA and ROS in response to GO treatment was found in shoots rather than roots. A similar phenomenon was also reported with maize plants where leaves were more sensitive than roots to the oxidative stress induced by sulfonated graphene; ${ }^{32}$ the MDA content in roots was increased only by the highest GO concentration $(500 \mathrm{mg} / \mathrm{L})$ while that in leaves was enhanced by GO concentrations ranging from 100 to $500 \mathrm{mg} / \mathrm{L}$. The underlying mechanism was explored in the following studies.

3.4. Alteration of the Uptake of Macro- and Microelements in Plants. To further examine the impact of GO 
and rGO on plant growth, we measured the uptake of several key nutrients that are essential for plant growth. rGO decreased the $\mathrm{Cu}$ level in plant tissues at $50 \mathrm{mg} / \mathrm{L}$ but showed no effects on the level of other elements (Figure S5); however, GO induced alteration of the levels of several elements including $\mathrm{P}$ and $\mathrm{Fe}$ in shoots and $\mathrm{Fe}$ and $\mathrm{Zn}$ in roots (Figure 4). Surprisingly, the Fe level in shoots $(415 \mathrm{mg} / \mathrm{kg})$ treated
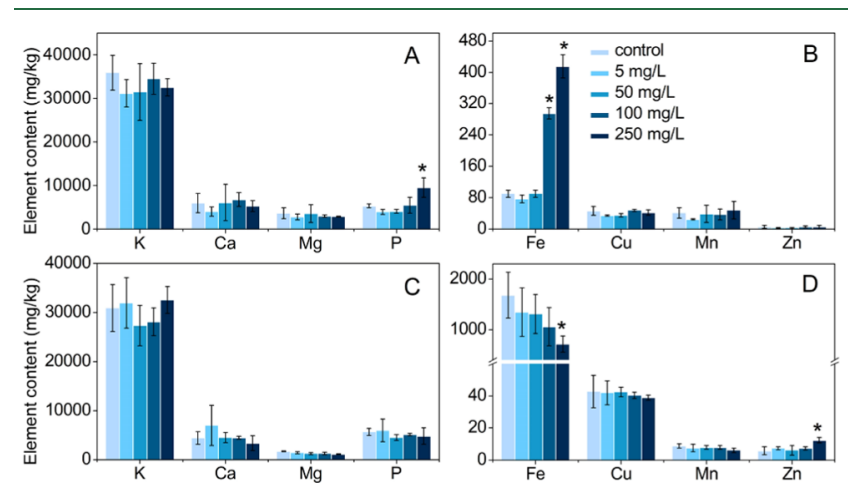

Figure 4. Macronutrient and micronutrient contents in shoots (A, B) and roots $(\mathrm{C}, \mathrm{D})$ of plants after exposure to different concentrations of GO for 3 weeks. * indicates significant difference compared with control at $P<0.05$.

with $250 \mathrm{mg} / \mathrm{L}$ GO was enhanced by 3.6 times as compared with that in the control plants $(90 \mathrm{mg} / \mathrm{kg})$. Rice plants usually maintain $60-300 \mathrm{mg} / \mathrm{kg}$ of Fe; when the Fe content exceeds $400 \mathrm{mg} / \mathrm{kg}$, the plant will experience toxicity due to $\mathrm{Fe}$ overload. ${ }^{33}$ The excessive $\mathrm{Fe}$ is translocated upward and accumulated in leaves, impairing the physiological processes of plants by generating ROS via the Haber-Weiss or Fenton reactions. ${ }^{34}$ In our study, the total $\mathrm{Fe}$ content in the GOexposed plants was not significantly changed (Figure S6A); however, the translocation of Fe from root to shoot was greatly enhanced (Figure S6B). The Fe level in shoots was increased up to $415 \mathrm{mg} / \mathrm{kg}$ by the $250 \mathrm{mg} / \mathrm{L}$ GO treatment, which is correlated with the overaccumulation of ROS and altered antioxidant enzymatic activities in plant leaves. These results suggested that GO-induced $\mathrm{Fe}$ overload and consequent oxidative stress in leaves is one possible mechanism causing the phytotoxicity observed. The increased P level (Figure 4A) was unlikely to be the driver of the toxicity because the highest $P$ level in the shoot $(9.5 \mathrm{mg} / \mathrm{g})$ was still below the concentration $(>13 \mathrm{mg} / \mathrm{g})$ at which $\mathrm{P}$ may become toxic to gramineous plants. ${ }^{35}$ Additionally, the toxicity in shoots occurred at $100 \mathrm{mg} / \mathrm{L}$ when there is no change of $\mathrm{P}$ levels, suggesting that $\mathrm{P}$ was not necessary for the occurrence of the toxicity.

3.5. Iron Overload Contributes More Than GO Per Se to the GO-Induced Phytotoxicity. Since both GO per se and Fe overload may induce oxidative stress in plants, a followup question is to understand the contributions of $\mathrm{GO}$ and $\mathrm{Fe}$ overload to the induced oxidative stress and phytotoxicity. In our study, the oxidative stress, lipid peroxidation, and overt phytotoxicity (reduction of biomass and seedling length) were found for leaves rather than roots; this pattern is similar to that found in Fe overloaded plants rather than graphene or other ENM treated plants. For example, it was reported that excessive $\mathrm{FeSO}_{4}$ treatment enhanced the MDA content in rice leaves by $134 \%$ while having no effect on the MDA levels in roots. ${ }^{36}$ While for ENMs, the roots are usually more sensitive than the leaves to ENM-induced toxicity, which might be due to the fact that most of the ENMs are adsorbed onto the root surface, while the upward translocation of ENMs is limited. ${ }^{37}$

Therefore, we deduced that excessive Fe uptake might be the main contributor to the toxicity found in this study rather than GO per se. To prove this hypothesis, we added an excessive amount of $\mathrm{Fe}\left(4 \mathrm{mM} \mathrm{FeSO}_{4}\right)$ to the $50 \mathrm{mg} / \mathrm{L} \mathrm{GO}$ suspension and examined the rice seedlings' growth. As compared with GO treatment alone, the dry weight of leaves was reduced by $27 \%$ (Figure $5 \mathrm{~A}$ ) and the MDA content in leaves was upregulated by $46 \%$ (Figure $5 \mathrm{~B}$ ) after exposure to $\mathrm{GO}+\mathrm{FeSO}_{4}$, which are correlated with a significantly enhanced $\mathrm{Fe}$ level in leaves (Figure $5 \mathrm{C}$ ). These results suggest that $\mathrm{Fe}$ overload contributed more than GO per se to the oxidative stress and subsequent toxicity in rice plants, although the effects of GO and rGO cannot be simply ignored since GO and $\mathrm{rGO}$ per se may generate ROS. ${ }^{38}$

3.6. Mechanisms Involved in the Overload of Fe in Leaves. Under anaerobic conditions, e.g., in paddy fields, $\mathrm{Fe}$ usually exists in the form of $\mathrm{Fe}^{2+}$, which is bioavailable for plant uptake. To avoid overaccumulation of $\mathrm{Fe}$, rice roots can release oxygen and oxidase to oxidize the $\mathrm{Fe}^{2+}$ to $\mathrm{Fe}^{3+}$, which precipitates to form a coating on the roots named "Fe plaque" ${ }^{33}$ The Fe plaque can prevent not only the uptake of excessive $\mathrm{Fe}^{2+}$ but also the entry of toxic heavy metals into plants. However, a decrease of $\mathrm{pH}$ can significantly promote the reduction of insoluble $\mathrm{Fe}^{3+}$ to $\mathrm{Fe}^{2+}$ that eventually leads to Fe toxicity, which affects a significant proportion of rice fields in many developing countries. ${ }^{33}$

The $\mathrm{pH}$ mediated $\mathrm{Fe}$ uptake not only applies for plants cultured in soil but also applies for hydroponic culture. It was also reported that low $\mathrm{pH}$ can significantly promote $\mathrm{Fe}^{2+}$ uptake by plants. ${ }^{39} \mathrm{We}$ found that GO acidified the nutrient solution while $\mathrm{rGO}$ did not change the $\mathrm{pH}$ significantly (Figure $6 \mathrm{~A})$. The $\mathrm{pH}$ of the $\mathrm{GO}$ suspensions decreased with increasing GO concentration. The $\mathrm{pH}$ values for 100 and $250 \mathrm{mg} / \mathrm{L} \mathrm{GO}$ in NS are 3.95 and 3.32, respectively, which are much lower than that of the normal NS ( $\mathrm{pH} 5.5)$. The low $\mathrm{pH}$ itself was unlikely the main reason for the toxicity based on two reasons: (1) rice is relatively tolerant of acidic conditions. Previous
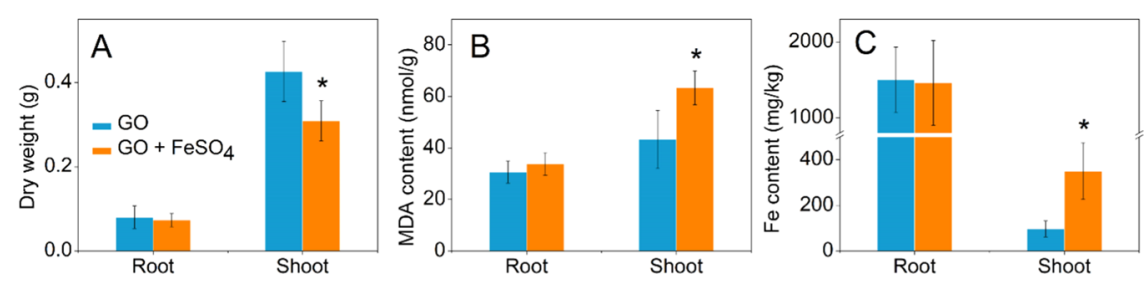

Figure 5. Dry weight (A), MDA content (B), and Fe content (C) in rice plants after exposure to GO (50 mg/L) and a mixture of GO (50 mg/L) and $\mathrm{FeSO}_{4}(4 \mathrm{mM})$ for 3 weeks. * indicates significant difference compared with GO treatment at $P<0.05$. 

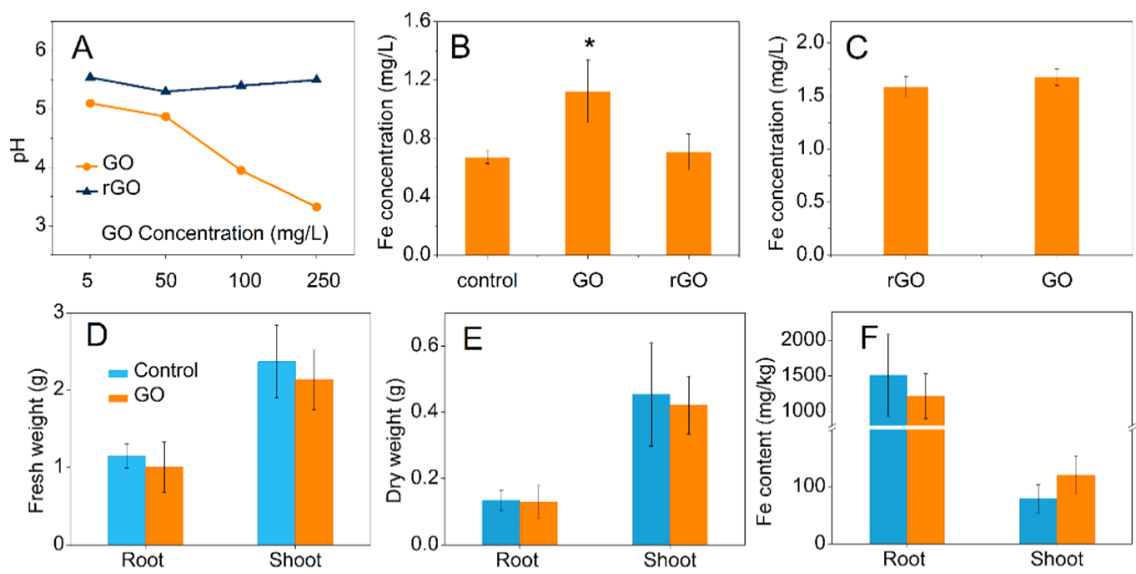

Figure 6. $\mathrm{pH}$ values of GO and rGO suspensions in nutrient solution (A), the free Fe concentration in collected xylem saps (B), and the free Fe concentration in nutrient solution after incubation with GO and rGO (C) from a starting concentration of $2.9 \mathrm{mg} / \mathrm{L}$. Fresh weight (D) and dry weight (E) of plant and $\mathrm{Fe}$ content $(\mathrm{F})$ in plant after exposure to $\mathrm{pH}$ adjusted $\mathrm{GO}$ suspension $(250 \mathrm{mg} / \mathrm{L}$, $\mathrm{pH} 5.5)$ for 3 weeks. * indicates significant difference compared with control at $P<0.05$.
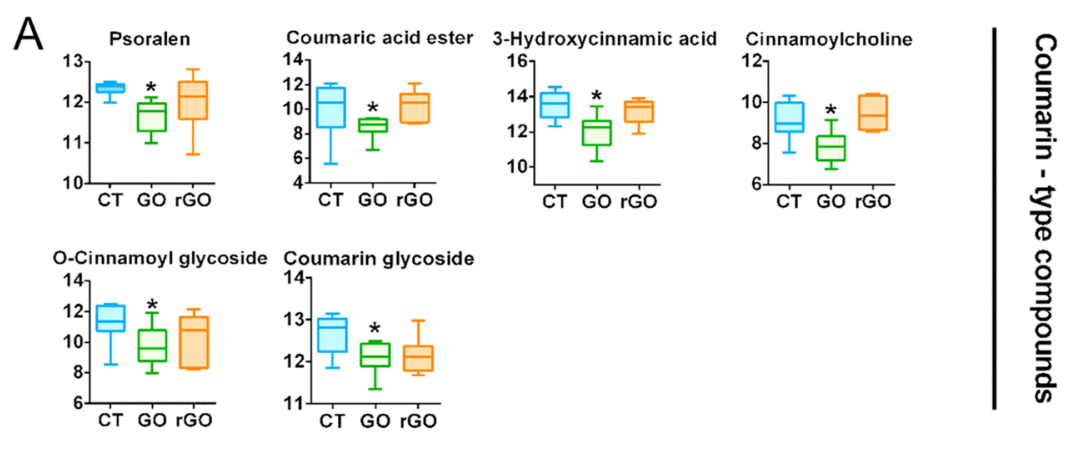

B
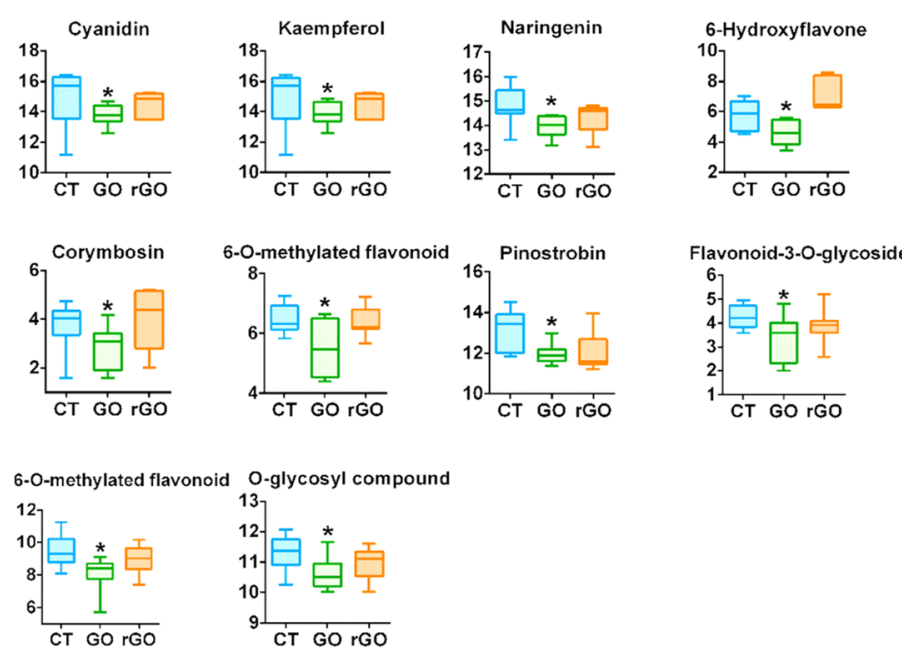

Figure 7. Box plots of relative abundance of coumarin-type compounds (A) and flavonoid-type compounds (B) in the leaves of rice treated with $250 \mathrm{mg} / \mathrm{L} \mathrm{GO}$ and $\mathrm{rGO}$ compared to the untreated controls $(n=8)$. * indicates significant difference compared with control.

studies showed that rice can grow normally at $\mathrm{pHs}$ as low as 3.4 , but the growth can be greatly impaired if Fe contents in the soil increased. ${ }^{40}$ (2) Impairment of the root growth should be also observed if $\mathrm{pH}$ was the reason for the toxicity. However, in our study, only the growth of shoots was impaired. Therefore, the low $\mathrm{pH}$ itself is not the main driver of the toxicity. Instead, the GO-induced decrease of $\mathrm{pH}$ can increase $\mathrm{Fe}$ mobilization and cause the observed Fe overload in the shoots. ${ }^{39}$ Figure $6 \mathrm{~B}$ further showed that the Fe content flux in the xylem sap was significantly enhanced by GO treatment.
Considering the high capacity for absorption of GBMs, which results from their high surface area, we further examined another possibility, which is that GO may enrich Fe on their surface by adsorption and then translocate to the leaves, which may enhance the Fe uptake. To do so, we compared the adsorption of Fe on GO and rGO (see method in Section 1, Supporting Information). The amount of $\mathrm{Fe}$ adsorbed onto $\mathrm{GO}$ and $\mathrm{rGO}$ was 1.22 and $1.31 \mathrm{mg} / \mathrm{L}$ (Figure 6C), respectively, which was nearly half of the $\mathrm{Fe}$ present in the nutrient solution $(2.9 \mathrm{mg} / \mathrm{L})$. We then estimated the amount 

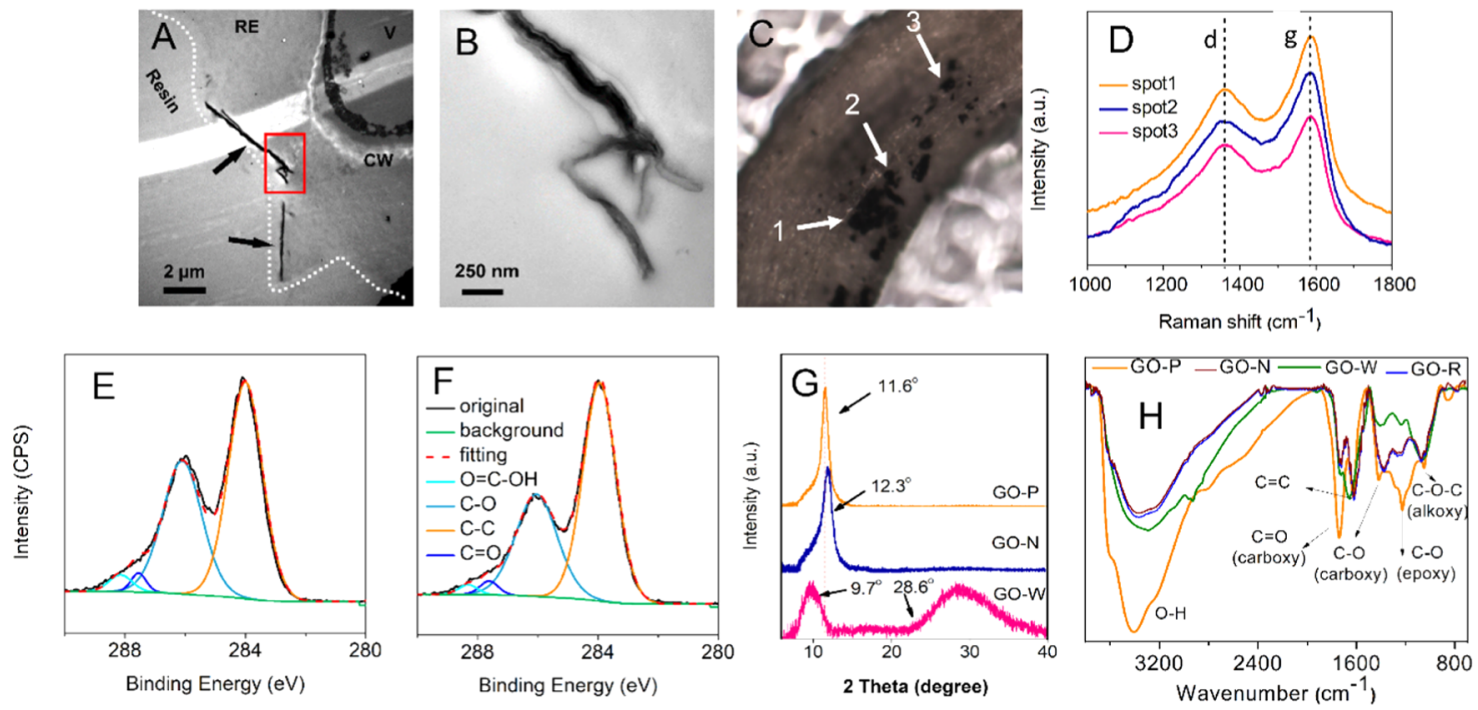

Figure 8. Characterization of GO after interaction with plants for 3 weeks. (A, B) TEM images of root sections; (B) is the magnified image of the rectangle area shown in (A). RE indicates the root exudate and CW indicates the cell wall. (C) Optical image of roots. (D) Raman spectra collected at the three spots shown in (C); $\mathrm{d}$ and g indicate the $\mathrm{d}$ band at $1363 \mathrm{~cm}^{-1}$ and g band at $1593 \mathrm{~cm}^{-1}$, respectively. The intensity ratios of $\mathrm{d}$ to $\mathrm{g}$, i.e., $I_{\mathrm{d}} / I_{\mathrm{g}}$ ratios, were 0.715 (spot 1), 0.723 (spot 2), and 0.783 (spot 3), respectively. (E) XPS spectra of GO-R (GO in residual NS after removal of plants). (F) Raman spectra of GO-W (GO washed off from roots). (G) XRD spectra of GO-P (pristine GO), GO-N (GO incubated in nutrient solution for 3 weeks), and GO-W. (H) FTIR spectra of GO-P, GO-N, GO-W, and GO-R.

of Fe that can be translocated with $\mathrm{GO}$ and $\mathrm{rGO}$ to the shoots (see the method for estimation of uptake in Section 1, Supporting Information). Only $0.098 \mathrm{mg} / \mathrm{kg}$ of $\mathrm{Fe}$ can be attributed to transport into the plants via adsorption onto GO or rGO. This is negligible compared with the total amount of $\mathrm{Fe}$ that was accumulated in the leaves $(325 \mathrm{mg} / \mathrm{kg})$, suggesting that the contribution of this mechanism to $\mathrm{Fe}$ overload is negligible.

When the $\mathrm{pH}$ of the $\mathrm{GO}$ suspension was adjusted back to normal $\mathrm{pH}$ (5.5) (see details of methods in Supporting Information), phytotoxicity was eliminated (Figure 6D,E) and the Fe content in shoots was normal (Figure 6F). These results provide solid evidence that $\mathrm{Fe}$ overload is the main cause of the GO-induced toxicity.

3.7. Plant Defense against Fe Overload via Reduced Production of Fe Transport Associated Metabolites. In our study, we found depressed production or excretion of ironmobilizing coumarin-type compounds and iron-chelating flavonoid-type compounds (Figure 7) in the leaves of GO treated plants, which may act as an important component of the iron depletion strategy in response to $\mathrm{Fe}$ overload. Coumarin-derived phenolics or their corresponding glycosides were all dramatically depressed in the GO treated plants. For example, psoralen, a linear furanocoumarin, was decreased by $36 \%$ (Figure $7 \mathrm{~A}$ ). The coumaric acid ester, 3-hydroxycinnamic acid, and cinnamoylcholine (a cinnamic acid ester), which are intermediates in the coumarin biosynthetic pathway, were significantly decreased by 78,62 , and $60 \%$, respectively. Glycosides, such as coumarin glycoside (4-methylumbelliferyl glucuronide) and cinnamoyl glycoside, were also significantly decreased. In addition, an array of flavonoid-derived compounds, including flavonoids (e.g., flavanones, flavones, flavonols, anthocyanidin), the methylated derivatives, and their glycosides were all downregulated in the GO-exposed plants (Figure 7B). For example, the concentrations of cyanidin (a type of anthocyanidin), kaempferol (a natural flavonol), naringenin (a flavanone), 6-hydroxyflavone (a flavone), and corymbosin (a flavone) were depressed by 70, 66, 48, 56, and $44 \%$, respectively. The decrement for other flavonoid derivatives ranged from 41 to $65 \%$. These data suggest that regulatory mechanisms at the metabolic level were evoked in leaves to sustain the iron homeostasis in response to GOinduced Fe overload.

3.8. Biotransformation of $\mathrm{GO}$ as a Pathway to Alleviate GO-Induced Phytotoxicity. ENMs may transform by interaction with plants, the process of which may determine their subsequent behavior, fate, and toxicity in plants. $\mathrm{CeO}_{2}$ ENMs are reported to be transformed in many plant species, ${ }^{41,42}$ being reduced and releasing $\mathrm{Ce}^{3+}$, which is found to be responsible for the toxicity of $\mathrm{CeO}_{2}$ ENMs to Lactuca plants. ${ }^{42}$ The released $\mathrm{Ce}^{3+}$ can bind with phosphates, which could be a detoxification process. ${ }^{43,44}$ Transformation of graphene materials has been reported in bacteria, ${ }^{28}$ plants, ${ }^{45}$ and in water under sunlight. ${ }^{46}$ Free radicals $\left(\mathrm{OH}^{\circ}\right)$ were reported to be involved in the transformation of graphene into $\mathrm{CO}_{2}$ in plant leaves, the process of which contributed to the elimination of graphene from plants following uptake and may thus reduce the graphene-induced phytotoxicity. ${ }^{45}$ Immobilization of root exudates onto GO and formation of ligand-GO complexes were also reported, which decreased the surface charge and increased the unpaired electrons and the toxicity of GO to zebrafish. ${ }^{47}$

We found that GO adsorbed onto the root surface, with a significant change of morphology from sheet to a folded shape (Figure 8A,B). A root exudate (RE) layer between the GO and the root epidermis cells can be observed, which might act as a barrier to prevent GO from entering the roots. Adsorption of GO onto the root surface was also clearly visible under the Raman microscope (Figure $8 \mathrm{C}$ ). The $I_{\mathrm{d}} / I_{\mathrm{g}}$ ratios $(0.715-$ 0.783) of the Raman spectra, collected from three spots on the roots (Figure $8 \mathrm{D}$ ), were significantly lower than that of pristine GO (0.98, Figure S7 and Table S4), suggesting that roots enhanced the disorder in the structure of $\mathrm{GO} .{ }^{47}$ The $\mathrm{O} / \mathrm{C}$ ratios of GO decreased significantly after interaction with 
plants (Figure 8E,F and Table S4), suggesting the partial transformation of GO into rGO. GO-W (which were washed from the root surface) showed a higher reduction degree $(\mathrm{O} /$ $\mathrm{C}, 0.31)$ than $\mathrm{GO}-\mathrm{R}(\mathrm{O} / \mathrm{C}, 0.4)$, suggesting that direct contact with the plant roots accelerated the reduction of GO. X-ray diffraction (XRD) analysis further confirmed the transformation of GO into rGO (Figure $8 \mathrm{G}$ ). Incubation in the nutrient solution (GO-N) only induced a slight shift of the (002) peak of GO from 11.6 to $12.3^{\circ}$, suggesting no alteration of the crystal structure but a decrease of the lattice spacing. However, interaction of GO with roots (GO-W) not only induced a shift of the (002) peak to $9.7^{\circ}$ but also led to the formation of a new peak at $28.6^{\circ}$, which is attributed to the (002) peak of $\mathrm{rGO}$, suggesting the reduction of GO. ${ }^{48}$ In agreement with the XPS and XRD results, FTIR showed that GO-W was reduced to a higher degree than GO-R, suggesting that contact of GO with plant roots facilitated the transformation of GO to rGO (Figure $8 \mathrm{H}$ ). The transformation of "toxic" GO into a relatively low-toxic "rGO" might act as a pathway to alleviate the toxicity of GO. The potential role of root-associated microbes in the transformation process remains to be explored.

Adsorption of rGO onto the root surface was also observed by TEM (Figure S9). FTIR spectra (Figure S10) showed an increased intensity of surface oxygen content after interaction with plant roots (rGO-W), suggesting partial oxidation of rGO. However, XRD analysis showed that the main peak (002) of rGO was not shifted (Figure S11), suggesting no changes to the crystal structure. The increased surface oxygen content observed by FTIR might be due to the adsorption of organic compounds from root exudates.

The present study reports for the first time a new mechanism of ENM-induced phytotoxicity, i.e., GO-induced $\mathrm{pH}$ alteration of nutrient solution and subsequent $\mathrm{Fe}$ overaccumulation and oxidative damage in plant leaves. Some previous studies have suggested that ENMs can disturb the macro- and microelement distribution in plants, however, a clear interpretation of these findings is lacking. The present study indicates that ENMs may cause toxicity to plants indirectly by altering the micronutrient uptake. The apparently different impact of GO and rGO on plant growth suggests that the phytotoxicity of GBMs is highly related to their surface oxygen content. The inconsistent use of GO or rGO with different surface oxygen densities might be one of the reasons that explain the inconsistency in the current literature. It should be noted that this is a short-term study carried out in a hydroponic condition. Effects of GBMs on plant in realistic soil environment over longer exposure time might be different and the mechanisms involved will be complicated by the soil components, which requires further studies.

\section{ASSOCIATED CONTENT}

\section{SI Supporting Information}

The Supporting Information is available free of charge at https://pubs.acs.org/doi/10.1021/acs.est.9b05794.

Experimental details and results; limit of detection, precision, and recovery data for ICP-OES for the selected elements; schematic illustration of xylem sap collection; size distribution of GO and $\mathrm{rGO} ; \zeta$-potential and hydrodynamic size of GO and rGO (PDF)

\section{AUTHOR INFORMATION}

\section{Corresponding Authors}

Peng Zhang - Key Laboratory for Biological Effects of Nanomaterials and Nanosafety, Institute of High Energy Physics, Chinese Academy of Sciences, Beijing 100049, China; School of Geography, Earth and Environmental Science, University of Birmingham, B15 2TT Birmingham, U.K.; 다이.org/0000-0002-2774-5534; Email: pengzhang@ ihep.ac.cn

Zhiyong Zhang - Key Laboratory for Biological Effects of Nanomaterials and Nanosafety, Institute of High Energy Physics, Chinese Academy of Sciences, Beijing 100049, China; 다이.org/0000-0001-9753-0873; Email: zhangzhy@ ihep.ac.cn

\section{Authors}

Zhiling Guo - School of Geography, Earth and Environmental Science, University of Birmingham, B15 2TT Birmingham, U.K.

Wenhe Luo - Key Laboratory for Biological Effects of Nanomaterials and Nanosafety, Institute of High Energy Physics, Chinese Academy of Sciences, Beijing 100049, China

Fazel Abdolahpur Monikh - Institute of Environmental Sciences (CML), Leiden University, 2300 RA Leiden, Netherlands; (1) orcid.org/0000-0001-9500-5303

Changiian Xie - Key Laboratory for Biological Effects of Nanomaterials and Nanosafety, Institute of High Energy Physics, Chinese Academy of Sciences, Beijing 100049, China

Eugenia Valsami-Jones - School of Geography, Earth and Environmental Science, University of Birmingham, B15 2TT Birmingham, U.K.

Iseult Lynch - School of Geography, Earth and Environmental Science, University of Birmingham, B15 2TT Birmingham, U.K.; 10 orcid.org/0000-0003-4250-4584

Complete contact information is available at: https://pubs.acs.org/10.1021/acs.est.9b05794

\section{Notes}

The authors declare no competing financial interest.

\section{ACKNOWLEDGMENTS}

This work was supported by Marie Skłodowska-Curie Individual Fellowships (NanoLabels Grant Agreement No. 750455 to P.Z.; NanoBBB Grant Agreement No. 798505 to Z.G.) under the European Union's Horizon 2020 research program.

\section{REFERENCES}

(1) Geim, A. K. Graphene: status and prospects. Science 2009, 324, $1530-1534$.

(2) Avouris, P. Graphene: electronic and photonic properties and devices. Nano Lett. 2010, 10, 4285-4294.

(3) Tonelli, F. M.; Goulart, V. A.; Gomes, K. N.; Ladeira, M. S.; Santos, A. K.; Lorençon, E.; Ladeira, L. O.; Resende, R. R. Graphenebased nanomaterials: biological and medical applications and toxicity. Nanomedicine 2015, 10, 2423-2450.

(4) Chabot, V.; Higgins, D.; Yu, A.; Xiao, X.; Chen, Z.; Zhang, J. A review of graphene and graphene oxide sponge: material synthesis and applications to energy and the environment. Energy Environ. Sci. 2014, 7, 1564-1596.

(5) Zhao, J.; Wang, Z.; White, J. C.; Xing, B. Graphene in the aquatic environment: adsorption, dispersion, toxicity and transformation. Environ. Sci. Technol. 2014, 48, 9995-10009. 
(6) Sanchez, V. C.; Jachak, A.; Hurt, R. H.; Kane, A. B. Biological interactions of graphene-family nanomaterials: an interdisciplinary review. Chem. Res. Toxicol. 2011, 25, 15-34.

(7) Gurunathan, S.; Arsalan Iqbal, M.; Qasim, M.; Park, C. H.; Yoo, H.; Hwang, J. H.; Uhm, S. J.; Song, H.; Park, C.; Do, J. T.; et al. Evaluation of Graphene Oxide Induced Cellular Toxicity and Transcriptome Analysis in Human Embryonic Kidney Cells. Nanomaterials 2019, 9, 969.

(8) Wang, Q.; Li, C.; Wang, Y.; Que, X. Phytotoxicity of Graphene Family Nanomaterials and Its Mechanisms: A Review Front. Chem. 2019, 7, Article 292.

(9) Kabiri, S.; Degryse, F.; Tran, D. N.; da Silva, R. C.; McLaughlin, M. J.; Losic, D. Graphene Oxide: A New Carrier for Slow Release of Plant Micronutrients. ACS Appl. Mater. Interfaces 2017, 9, 4332543335 .

(10) Zhang, M.; Gao, B.; Chen, J.; Li, Y.; Creamer, A. E.; Chen, H. Slow-release fertilizer encapsulated by graphene oxide films. Chem. Eng. J. 2014, 255, 107-113.

(11) Andelkovic, I. B.; Kabiri, S.; Tavakkoli, E.; Kirby, J. K.; McLaughlin, M. J.; Losic, D. Graphene oxide-Fe (III) composite containing phosphate-A novel slow release fertilizer for improved agriculture management. J. Cleaner Prod. 2018, 185, 97-104.

(12) Ren, W.; Ren, G.; Teng, Y.; Li, Z.; Li, L. Time-dependent effect of graphene on the structure, abundance, and function of the soil bacterial community. J. Hazard. Mater. 2015, 297, 286-294.

(13) Chung, H.; Kim, M. J.; Ko, K.; Kim, J. H.; Kwon, H.-a.; Hong, I.; Park, N.; Lee, S.-W.; Kim, W. Effects of graphene oxides on soil enzyme activity and microbial biomass. Sci. Total Environ. 2015, 514, 307-313.

(14) Kim, M.-J.; Ko, D.; Ko, K.; Kim, D.; Lee, J.-Y.; Woo, S. M.; Kim, W.; Chung, $\mathrm{H}$. Effects of silver-graphene oxide nanocomposites on soil microbial communities. J. Hazard. Mater. 2018, 346, 93-102.

(15) Dong, S.; Xia, T.; Yang, Y.; Lin, S.; Mao, L. Bioaccumulation of 14C-labeled graphene in an aquatic food chain through direct uptake or trophic transfer. Environ. Sci. Technol. 2018, 52, 541-549.

(16) Begum, P.; Ikhtiari, R.; Fugetsu, B. Graphene phytotoxicity in the seedling stage of cabbage, tomato, red spinach, and lettuce. Carbon 2011, 49, 3907-3919.

(17) Zhang, P.; Zhang, R.; Fang, X.; Song, T.; Cai, X.; Liu, H.; Du, S. Toxic effects of graphene on the growth and nutritional levels of wheat (Triticum aestivum L.): short-and long-term exposure studies. J. Hazard. Mater. 2016, 317, 543-551.

(18) Cheng, F.; Liu, Y.-F.; Lu, G.-Y.; Zhang, X.-K.; Xie, L.-L.; Yuan, C.-F.; Xu, B.-B. Graphene oxide modulates root growth of Brassica napus L. and regulates $\mathrm{ABA}$ and IAA concentration. J. Plant Physiol. 2016, 193, 57-63.

(19) Chen, L.; Wang, C.; Li, H.; Qu, X.; Yang, S.-T.; Chang, X.-L. Bioaccumulation and toxicity of 13C-skeleton labeled graphene oxide in wheat. Environ. Sci. Technol. 2017, 51, 10146-10153.

(20) Zhou, Q.; Hu, X. Systemic stress and recovery patterns of rice roots in response to graphene oxide nanosheets. Environ. Sci. Technol. 2017, 51, 2022-2030.

(21) Servin, A. D.; White, J. C. Nanotechnology in agriculture: next steps for understanding engineered nanoparticle exposure and risk. NanoImpact 2016, 1, 9-12.

(22) Hu, X.; Zhou, Q. Novel hydrated graphene ribbon unexpectedly promotes aged seed germination and root differentiation. Sci. Rep. 2015, 4, No. 3782.

(23) He, Y.; Hu, R.; Zhong, Y.; Zhao, X.; Chen, Q.; Zhu, H. Graphene oxide as a water transporter promoting germination of plants in soil. Nano Res. 2018, 11, 1928-1937.

(24) Zhang, P.; Ma, Y.; Xie, C.; Guo, Z.; He, X.; Valsami-Jones, E.; Lynch, I.; Luo, W.; Zheng, L.; Zhang, Z. Plant species-dependent transformation and translocation of ceria nanoparticles. Environ. Sci.: Nano 2019, 6, 60-67.

(25) Wang, Q.; Zhao, S.; Zhao, Y.; Rui, Q.; Wang, D. Toxicity and translocation of graphene oxide in Arabidopsis plants under stress conditions. RSC Adv. 2014, 4, 60891-60901.
(26) Cui, D.; Zhang, P.; Ma, Y.; He, X.; Li, Y.; Zhang, J.; Zhao, Y.; Zhang, Z. Effect of cerium oxide nanoparticles on asparagus lettuce cultured in an agar medium. Environ. Sci.: Nano 2014, 1, 459-465.

(27) De Jesus, L. R.; Dennis, R. V.; Depner, S. W.; Jaye, C.; Fischer, D. A.; Banerjee, S. Inside and outside: X-ray absorption spectroscopy mapping of chemical domains in graphene oxide. J. Phys. Chem. Lett. 2013, 4, 3144-3151.

(28) Guo, Z.; Xie, C.; Zhang, P.; Zhang, J.; Wang, G.; He, X.; Ma, Y.; Zhao, B.; Zhang, Z. Toxicity and transformation of graphene oxide and reduced graphene oxide in bacteria biofilm. Sci. Total Environ. 2017, 580, 1300-1308.

(29) Liu, L.; Zhu, C.; Fan, M.; Chen, C.; Huang, Y.; Hao, Q.; Yang, J.; Wang, H.; Sun, D. Oxidation and degradation of graphitic materials by naphthalene-degrading bacteria. Nanoscale 2015, 7, 13619-13628.

(30) Zhang, P.; He, X.; Ma, Y.; Lu, K.; Zhao, Y.; Zhang, Z. Distribution and bioavailability of ceria nanoparticles in an aquatic ecosystem model. Chemosphere 2012, 89, 530-535.

(31) Wang, J.; Wei, Y.; Shi, X.; Gao, H. Cellular entry of graphene nanosheets: the role of thickness, oxidation and surface adsorption. RSC Adv. 2013, 3, 15776-15782.

(32) Ren, W.; Chang, H.; Teng, Y. Sulfonated graphene-induced hormesis is mediated through oxidative stress in the roots of maize seedlings. Sci. Total Environ. 2016, 572, 926-934.

(33) Mahender, A.; Swamy, B.; Anandan, A.; Ali, J. Tolerance of iron-deficient and-toxic soil conditions in rice. Plants 2019, 8, 31.

(34) Kehrer, J. P. The Haber-Weiss reaction and mechanisms of toxicity. Toxicology 2000, 149, 43-50.

(35) Shane, M. W.; McCully, M. E.; Lambers, H. Tissue and cellular phosphorus storage during development of phosphorus toxicity in Hakea prostrata (Proteaceae). J. Exp. Bot. 2004, 55, 1033-1044.

(36) Müller, C.; Kuki, K. N.; Pinheiro, D. T.; de Souza, L. R.; Silva, A. I. S.; Loureiro, M. E.; Oliva, M. A.; Almeida, A. M. Differential physiological responses in rice upon exposure to excess distinct iron forms. Plant Soil 2015, 391, 123-138.

(37) Zhang, P.; Ma, Y.; Zhang, Z. Interactions between Engineered Nanomaterials and Plants: Phytotoxicity, Uptake, Translocation, and Biotransformation. In Nanotechnology and Plant Sciences; Springer, 2015; pp 77-99.

(38) Liu, S.; Zeng, T. H.; Hofmann, M.; Burcombe, E.; Wei, J.; Jiang, R.; Kong, J.; Chen, Y. Antibacterial activity of graphite, graphite oxide, graphene oxide, and reduced graphene oxide: membrane and oxidative stress. ACS Nano 2011, 5, 6971-6980.

(39) Zhao, T.; Ling, H. Q. Effects of $\mathrm{pH}$ and nitrogen forms on expression profiles of genes involved in iron homeostasis in tomato. Plant, Cell Environ. 2007, 30, 518-527.

(40) Tanaka, A.; Navasero, S. Growth of the rice plant on acid sulfate soils. Soil Sci. Plant Nutr. 1966, 12, 23-30.

(41) Zhang, P.; Ma, Y.; Zhang, Z.; He, X.; Zhang, J.; Guo, Z.; Tai, R.; Zhao, Y.; Chai, Z. Biotransformation of ceria nanoparticles in cucumber plants. ACS Nano 2012, 6, 9943-9950.

(42) Zhang, P.; Ma, Y.; Zhang, Z.; He, X.; Li, Y.; Zhang, J.; Zheng, L.; Zhao, Y. Species-specific toxicity of ceria nanoparticles to Lactuca plants. Nanotoxicology 2015, 9, 1-8.

(43) Li, R.; Ji, Z.; Chang, C. H.; Dunphy, D. R.; Cai, X.; Meng, H.; Zhang, H.; Sun, B.; Wang, X.; Dong, J.; et al. Surface interactions with compartmentalized cellular phosphates explain rare earth oxide nanoparticle hazard and provide opportunities for safer design. ACS Nano 2014, 8, 1771-1783.

(44) Briffa, S. M.; Lynch, I.; Hapiuk, D.; Valsami-Jones, E. Physical and chemical transformations of zirconium doped ceria nanoparticles in the presence of phosphate: Increasing realism in environmental fate and behaviour experiments. Environ. Pollut. 2019, 974-981.

(45) Huang, C.; Xia, T.; Niu, J.; Yang, Y.; Lin, S.; Wang, X.; Yang, G.; Mao, L.; Xing, B. Transformation of 14C-Labeled Graphene to $14 \mathrm{CO} 2$ in the Shoots of a Rice Plant. Angew. Chem. 2018, 130, 99079911.

(46) Hou, W.-C.; Chowdhury, I.; Goodwin, D. G., Jr.; Henderson, W. M.; Fairbrother, D. H.; Bouchard, D.; Zepp, R. G. Photochemical 
transformation of graphene oxide in sunlight. Environ. Sci. Technol. 2015, 49, 3435-3443.

(47) Du, J.; Hu, X.; Mu, L.; Ouyang, S.; Ren, C.; Du, Y.; Zhou, Q. Root exudates as natural ligands that alter the properties of graphene oxide and environmental implications thereof. RSC Adv. 2015, 5, $17615-17622$.

(48) Huang, H.-H.; De Silva, K. K. H.; Kumara, G.; Yoshimura, M. Structural evolution of hydrothermally derived reduced graphene oxide. Sci. Rep. 2018, 8, No. 6849. 